\title{
Novel analysis of prognosis of young patients with stage II differentiated thyroid cancer based on AJCC 8.0 and 6.0 criteria to implement the staging system
}

\author{
Yihui Huang ${ }^{1}$, Ling Zhou ${ }^{1}$, Wen Zeng ${ }^{2}$, Sichao Chen ${ }^{1}$, Wei Zhou ${ }^{1}$, Wei Wei ${ }^{3}$, Chao Zhang ${ }^{4}$, Di Hu ${ }^{1}$, \\ Min Wang ${ }^{1}$, Zeming Liu ${ }^{1 *}$, Liang Guo ${ }^{1 *}$ \\ ${ }^{1}$ Department of Plastic Surgery, Zhongnan Hospital of Wuhan University, Wuhan, China; ${ }^{2}$ Department of Ophthalmology, Zhongnan Hospital \\ of Wuhan University, Wuhan, China; ${ }^{3}$ Department of Pediatrics, St John Hospital and Medical Center, Detroit, MI, USA; ${ }^{4}$ Department of \\ Cardiovascular Surgery, Union Hospital, Tongji Medical College, Huazhong University of Science and Technology, Wuhan, China \\ Contributions: (I) Conception and design: Y Huang, M Wang; (II) Administrative support: Z Liu, L Guo; (III) Provision of study materials or patients: \\ L Zhou, W Zhou; (IV) Collection and assembly of data: W Zeng, W Wei; (V) Data analysis and interpretation: S Chen, C Zhang, D Hu; (VI) \\ Manuscript writing: All authors; (VII) Final approval of manuscript: All authors. \\ "These authors contributed equally to this work. \\ Correspondence to: Liang Guo; Zeming Liu. Zhongnan Hospital of Wuhan University, Donghu Road 169, Wuchang District, Wuhan 430071, China. \\ Email: guoliangwhzn@163.com; 6myt@163.com.
}

Background: The incidence of thyroid cancer among young adults is increasing; however, the clinical challenges specific to this population, such as diagnosis, reduced healthcare access, and inconsistent care, have received limited attention. Here, we conducted a subgroup analysis on a series of relatively young patients with differentiated thyroid carcinomas (DTCs), focusing on those with distant metastases at stage II, to obtain a deeper understanding of the factors influencing survival.

Methods: Information on $<45$ - or $<55$-year-old patients at any $\mathrm{T} / \mathrm{N}$ stage with distant metastasis (M1) was extracted from the SEER database according to the staging system in the $6^{\text {th }}$ and $8^{\text {th }}$ American Joint Committee on Cancer (AJCC) editions, respectively. Patient mortality was evaluated using Cox proportional hazards regression analyses and Kaplan-Meier analyses with log-rank tests.

Results: Both cancer-specific and all-cause mortality rates per 1,000 person-years for patients $\geq 35$ years old significantly differed from those of patients $<35$ years old. DTC-specific survival curves also significantly differed between these age groups, according to both the AJCC 6.0 and 8.0-based analyses $(\mathrm{P}=0.0017$ and $\mathrm{P}<0.001$, respectively), as did patient survival curves $(\mathrm{P}=0.0003, \mathrm{P}<0.001$, respectively). The multivariate Cox regression model also revealed that poor OS was strongly predicted by race $(\mathrm{P}<0.001)$ in the analysis based on the criteria of $8^{\text {th }}$ AJCC staging system.

Conclusions: Age is a risk factor for disease-specific and overall survival (OS) in young patients with stage II DTC, and young male patients exhibited poorer survival than females. Race also emerged as a potential risk factor for young patients in stage II. These findings offer guidance for improving the older and newer versions of the AJCC staging system.

Keywords: Thyroid cancer; stage II; distant metastasis; prognosis; Surveillance, Epidemiology, and End Results (SEER)

Submitted Jan 04, 2020. Accepted for publication Aug 28, 2020.

doi: $10.21037 / \mathrm{gs}-20-46$

View this article at: http://dx.doi.org/10.21037/gs-20-46 


\section{Introduction}

Differentiated thyroid carcinomas (DTCs), comprising papillary thyroid carcinoma (PTC) and follicular thyroid carcinoma (FTC), are widely known as indolent diseases, and their incidence has been increasing more rapidly than that of any other cancer in the US $(1,2)$. As DTCs are generally associated with high overall survival (OS) rates, they are expected to become the third most common cancer among females by 2019 (3,4). According to recent population study, the incidence of thyroid cancer has increased by an average of $3.6 \%$ per year in the latest 45 years $(5,6)$. In addition, the most common carcinoma in American adults 16-33 years of age at present is thyroid carcinoma (7). Despite the increased morbidity and incidence of thyroid carcinomas among adolescents and young adults, relatively limited attention has been paid to the specific challenges, including diagnosis, reduced health care access, and inconsistent care, that face this group of patients.

The prognosis of DTC is, to a certain degree, dependent on age, the presence of distant metastases, and the extent and size of the primary tumor (2). Among these relevant factors, the biological characteristics of DTC are most highly dependent on age, with young patients showing a much greater survival rate than older patients (8). Indeed, DTC is the only human malignant tumor for which age is included as part of the American Joint Committee on Cancer/Union for International Cancer Control (AJCC/ UICC) staging system (1). In the eighth edition of the AJCC/tumor, lymph node, metastasis (TNM) cancer staging system, which was officially implemented on January 1, 2018, the age cut-off was raised from 45 years in the older AJCC/TNM cancer staging systems (up to and including the 7 th edition) to 55 years (9). Besides the difference in the cut-off point in age, other differences between $6^{\text {th }}$ and $8^{\text {th }}$ edition of AJCC/TNM cancer staging systems including $\mathrm{T}$-stage, $\mathrm{N}$-stage and staging criteria for older patients ( $\geq 45 / 55$ years old). The decision for using the age of 45 years as a distinct cut-off point was mainly based on data from the mid-1900s, when a sharp increase in thyroid cancer mortality starting at around the age of 40-50 years was demonstrated (1). Despite this recent switch from the age of 45 years to that of 55 years as the cut-off point, few adjustments have been made to the staging system for young patients. Specifically, all young patients without distant metastases are classified at stage I regardless of tumor size, lymph node status, or extrathyroidal extension. Young patients ( $<45 / 55$ years old) are only classified at stage
II when they have distant metastases. By contrast, older patients are classified from stages I through IVc, reflecting the fact that younger age can dampen the deleterious effects generally associated with advanced disease to some extent (2). Poor survival in patients with DTC who present with metastatic disease is widely accepted (10). However, as patients $<45 / 55$ years old with distant metastases appear to exhibit poorer prognosis than patients in the same age group at stage I, a more thorough understanding of the relationship between the relevant factors and survival in young patients with DTC is needed, especially for those with distant metastases (stage II). In addition, it is necessary to determine the impact that this relationship has on patient outcomes.

In the present study, we conducted a subgroup analysis on a series of patients with DTC at stage II (with distant metastases), who were $<45 / 55$ years old, from the Surveillance, Epidemiology, and End Results (SEER) database. The objective of the study was to identify and determine the influence of several risk factors, including age, sex, race, histological type, TN-stage, extension status, radiation treatment, or surgery type, on the prognosis of young patients with stage II DTC with the aim of improving diagnosis and disease management among this group. We present the following article in accordance with the STROBE reporting checklist (available at http://dx.doi. org/10.21037/gs-20-46).

\section{Methods}

\section{Data sources}

The study cohort of patients with DTC was recruited from the SEER database, which is supported by the National Cancer Institute and Centers for Disease Control and Prevention; data collected between 2004 and 2013 were used (11). This study conformed to the provisions of the Declaration of Helsinki (as revised in 2013). The SEER database contains information on morbidity, prevalence, mortality, and population-based variables for patients worldwide, as well as details of the primary characteristics of the tumor, including site, spread, and histology where available, along with limited information regarding treatment, excluding chemotherapy (12).

\section{AfCC staging and data preparation}

Since SEER is a publicly available database with 
anonymized data, no ethical review was required. We examined SEER data collected between 2004 and 2013 and selected patients with a diagnosis of DTC, defined by a combination of the ICD-10 site code of C73.9 (i.e., thyroid) and diagnostic codes of papillary and/or follicular histology, including "papillary carcinoma," "papillary adenocarcinoma," "oxyphilic adenocarcinoma", "follicular adenocarcinoma," "papillary \& follicular adenocarcinoma," and "papillary cyst-adenocarcinoma." Patients with missing or unknown data regarding follow-up were excluded from analysis. A total of 99,015 cases were enrolled in this research.

The SEER system indicates disease stages using AJCC 6.0 for data from 2004 to 2013. At the time of this analysis, the new $8^{\text {th }}$ AJCC edition had not been implemented; therefore, we applied the AJCC 8.0 criteria to restage all cases of DTC in the SEER dataset 18 from 2004 to 2013 with available TNM information. The selection process is described in detail under Supplementary. We extracted information for patients at any $\mathrm{T} / \mathrm{N}$ stage with distant metastasis (M1) who were $<45$ years of age, based on the staging system of the $6^{\text {th }}$ AJCC edition, as well as for patients at any T/N stage with distant metastasis (M1) who were $<55$ years of age, using the staging system of the $8^{\text {th }}$ AJCC edition for further comparison.

\section{Statistical analyses}

According to Oyer et al. (13), an increase in age was associated with a significant decrease in disease-specific survival (DSS) for each decade. Specifically, patients who were 35-44 years of age showed a statistically significant decrease in DSS compared with those that were 25-34 years old. Based on these findings, we chose 35 years of age as a cut-off point to regroup patients $<45 / 55$ years old $(<45$ or $<55$ years old according to the $6^{\text {th }}$ and $8^{\text {th }}$ editions, respectively) with distant metastases for survival analyses. The follow-up data for selected patients were evaluated to calculate the rates of cancer-specific survival (CSS) and OS, as well as the mortality rates per 1,000 person-years. In addition, the mortality rates per 1,000 person-years among different groups were compared, with consideration of relevant factors. Hazard ratios (HRs) were used to determine the magnitude of the effect of disease stage on DTC-specific patient mortality, and $95 \%$ confidence intervals (CIs) were used to indicate the significance of the risk. Univariate and multivariate Cox regression analyses were performed to examine whether DSS and OS were associated with age, sex, race, histological type, TNM stage, extension status, radiation treatment, or surgery type. Patient survival curves were analyzed by Kaplan-Meier analyses with log-rank tests, and with Cox proportional hazards regression analyses.

\section{Results}

\section{General findings}

The distribution of patients in each stage classified according to the $6^{\text {th }}$ and $8^{\text {th }}$ AJCC staging system is summarized in Table S1. The proportion of patients classified in stage II based on the criteria of the $6^{\text {th }}$ edition $(7.64 \%)$ and $8^{\text {th }}$ edition $(7.54 \%)$ was similar. Moreover, there was a greater risk in younger patients $(<45$ or $<55$ years old according to the $6^{\text {th }}$ and $8^{\text {th }}$ editions, respectively) than in those $\geq 45$ or $\geq 55$ years old (Table S2). The flow diagram in Figure 1 shows the total number of patients extracted from the database, how all of the patients were categorized, and how many patients were excluded from analysis under the staging system in the $6^{\text {th }}$ and $8^{\text {th }}$ AJCC editions. Overall, there were 276 patients in the $<45$-year-old group and 461 patients in the $<55$-year-old group. The other demographic characteristics of these two groups are shown in Table 1.

\section{Clinicopathological parameters that affect CSS and OS}

Univariate Cox regression analyses revealed that the CSS of patients classified according to both the $6^{\text {th }}$ and $8^{\text {th }}$ editions of the AJCC staging systems was significantly associated with age at diagnosis $(\mathrm{P}=0.01, \mathrm{P}<0.001$, respectively), sex $(\mathrm{P}=0.006, \mathrm{P}=0.001)$, histological type $(\mathrm{P}=0.04, \mathrm{P}=0.001)$, and T-stage $(\mathrm{P}=0.008, \mathrm{P}<0.001)$ (Table 2); a similar result was observed for OS (Table 3). Patients who were $\geq 35$ years old $(\mathrm{P}=0.001, \mathrm{P}<0.001)$, at stage $\mathrm{T} 4(\mathrm{P}=0.002, \mathrm{P}<0.001)$, or male $(\mathrm{P}=0.018, \mathrm{P}<0.001)$ exhibited poorer $\mathrm{OS}$ under both staging systems. However, multivariate Cox regression analyses showed that only age at diagnosis and race were associated with CSS under the $8^{\text {th }}$ edition staging system, in which patients diagnosed at $\geq 35$ years old $(\mathrm{P}=0.001)$ and black patients $(\mathrm{P}<0.001)$ had significantly poorer CSS (Table 4 and Table S3). The multivariate Cox regression model also revealed that poor OS was predicted by age $(\mathrm{P}=0.003)$ and surgery type $(\mathrm{P}=0.007)$ based on analysis using the $6^{\text {th }}$ AJCC edition, and most strongly predicted by age $(\mathrm{P}<0.001)$, sex $(\mathrm{P}=0.013)$, and race $(\mathrm{P}<0.001)$ based on 


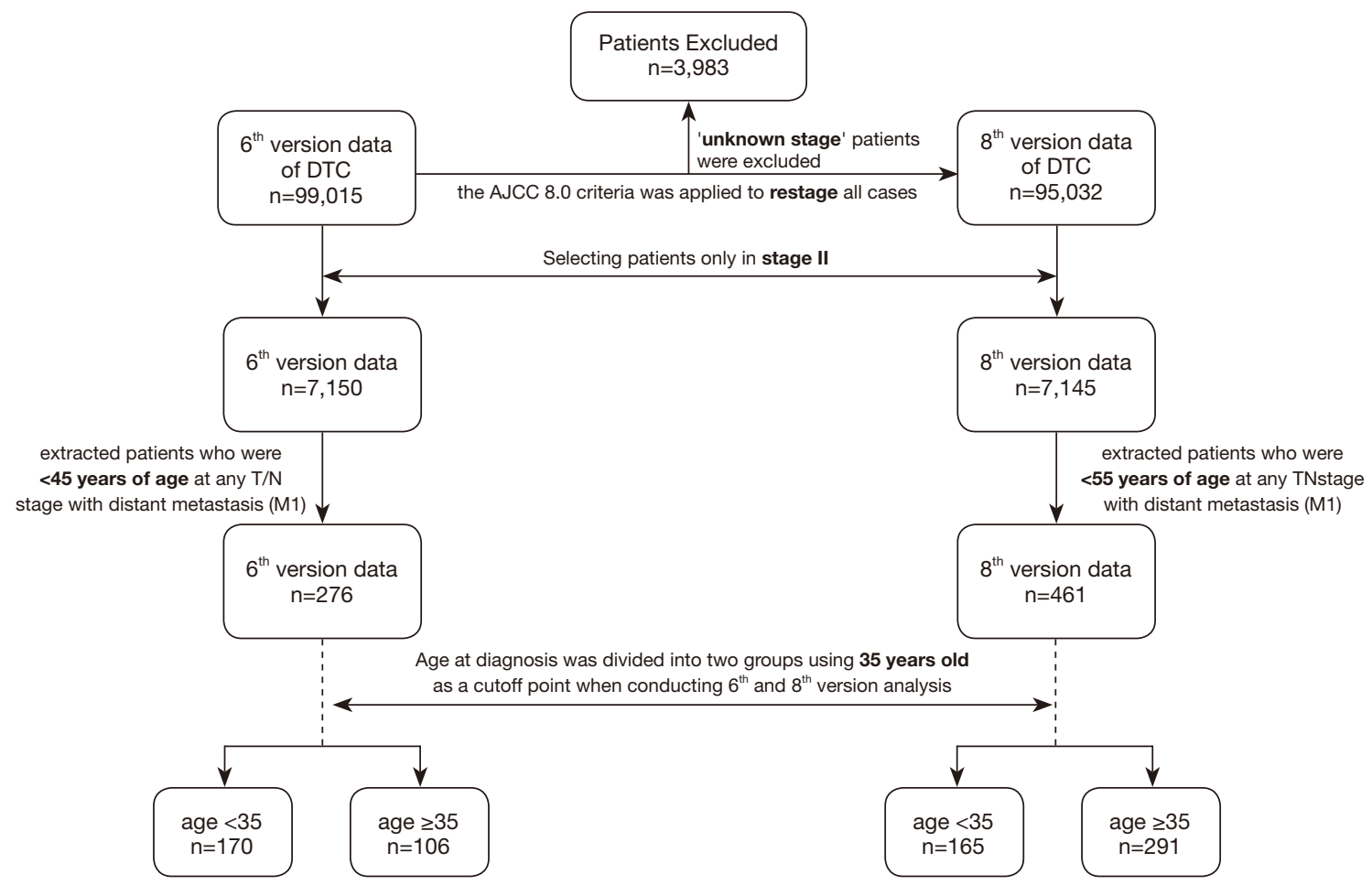

Figure 1 The flow diagram of the selection process, showing the total number of patients extracted from the database and how many patients were excluded from analysis under the staging system in the $6^{\text {th }}$ and $8^{\text {th }}$ AJCC editions (five patients lacked the information of age in the $8^{\text {th }}$ version data).

analysis using the $8^{\text {th }}$ edition of the AJCC (Table 5).

\section{Cancer-specific and all-cause mortality rates}

During the follow-up period, substantially higher rates of cancer-specific deaths and all-cause mortality deaths were observed under the AJCC 8.0-based analysis than under the AJCC 6.0-based analysis (Table 6). Given the associations of age at diagnosis, sex, race, and surgery type with CSS and OS based on the univariate and multivariate Cox analyses described above, we further calculated the cancer-specific and all-cause mortality rates in different groups based on these factors. As shown in Table 7, both the cancer-specific and all-cause mortality rates per 1,000 person-years of patients aged $\geq 35$ years significantly differed from those of patients $<35$ years old.

\section{Patient and DTC-specific survival curves}

Kaplan-Meier analyses revealed a significant difference in the DTC-specific survival curves between patients diagnosed at $<35$ and $\geq 35$ years old under both the AJCC 6.0 and 8.0 -based analyses $(\mathrm{P}=0.0017$ and $\mathrm{P}<0.0001$, respectively; Figure $2 A, B)$. However, there was no difference in DTC-specific survival curves according to race according to the AJCC 6.0-based analysis ( $\mathrm{P}=0.1178$, Figure 3): all curves were flat, with only a minimal decline. A similar difference between age groups was found for patient survival curves $\left(6^{\text {th }}\right.$ edition $\mathrm{P}=0.0003,8^{\text {th }}$ edition $\mathrm{P}<0.0001$; Figure $4 A, B)$. However, the decline of the patient survival curve in both analyses was sharper for male patients than for female patients $\left(6^{\text {th }}\right.$ edition $\mathrm{P}=0.0159$, Figure $5 A$; $8^{\text {th }}$ edition $\mathrm{P}=0.0003$, Figure $5 B$ ). Similar to the DTC-specific curves, there was no influence of race on patient survival curves ( $\mathrm{P}=0.2069$, Figure 6). Moreover, the patient survival curves did not differ significantly between surgery types according to either the AJCC 6.0-based analysis $(\mathrm{P}=0.7050)$ or AJCC 8.0-based analysis $(\mathrm{P}=0.4334)$ (Figure $7 A, B)$.

\section{Discussion}

Papillary and follicular carcinomas are DTCs that account 
Table 1 Characteristics of patients at any T/N stage with distant metastasis (M1) and were $<45$ years of age according to the staging system of the $6^{\text {th }} \mathrm{AJCC}$ edition, as well as patients at any T/N stage with distant metastasis (M1) who were $<55$ years of age according to the staging system of the $8^{\text {th }}$ AJCC edition (the proportion is presented with effective percentage)

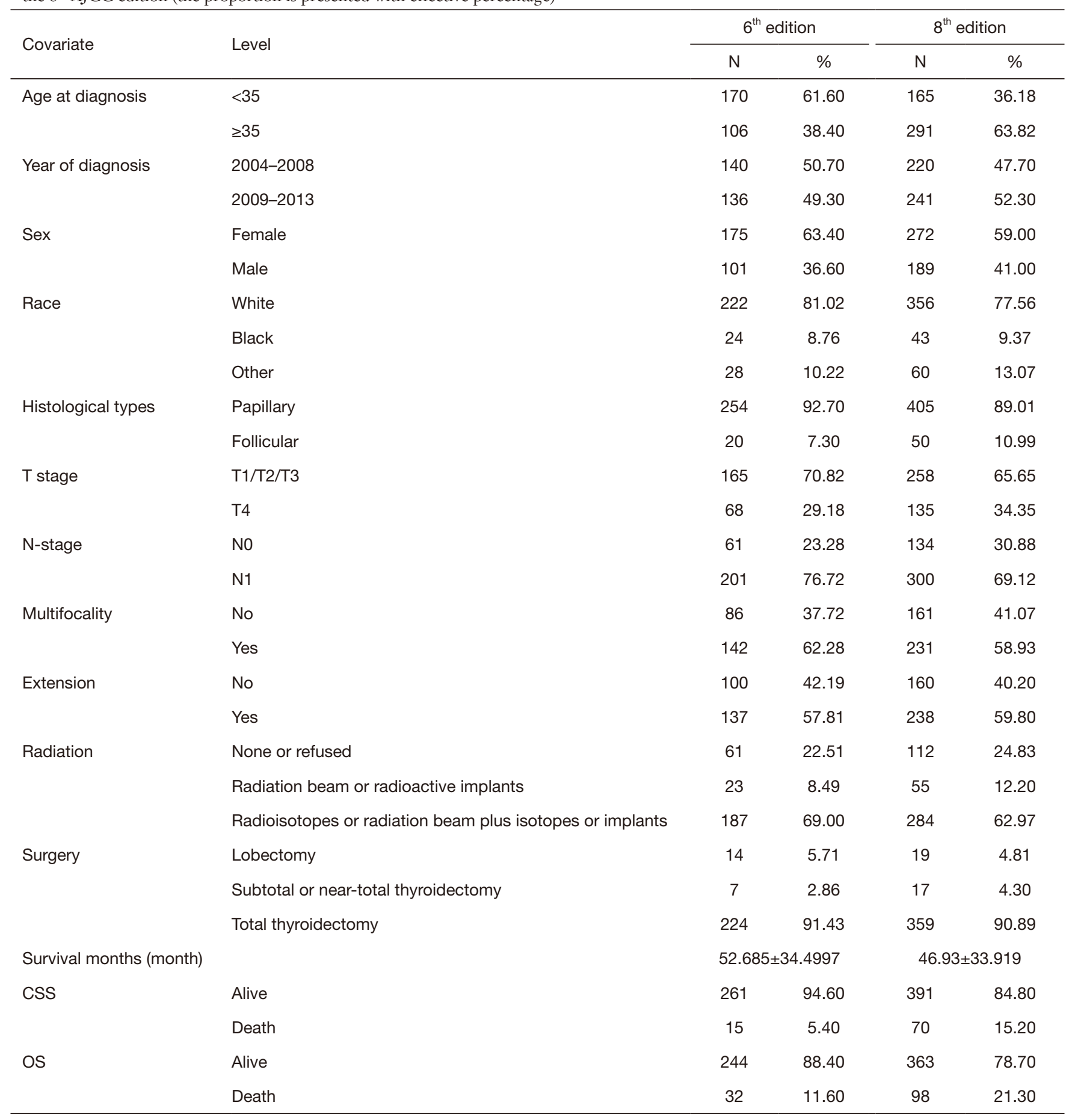


Table 2 Univariate analyses results for clinicopathologic parameters associated with the cancer-specific survival

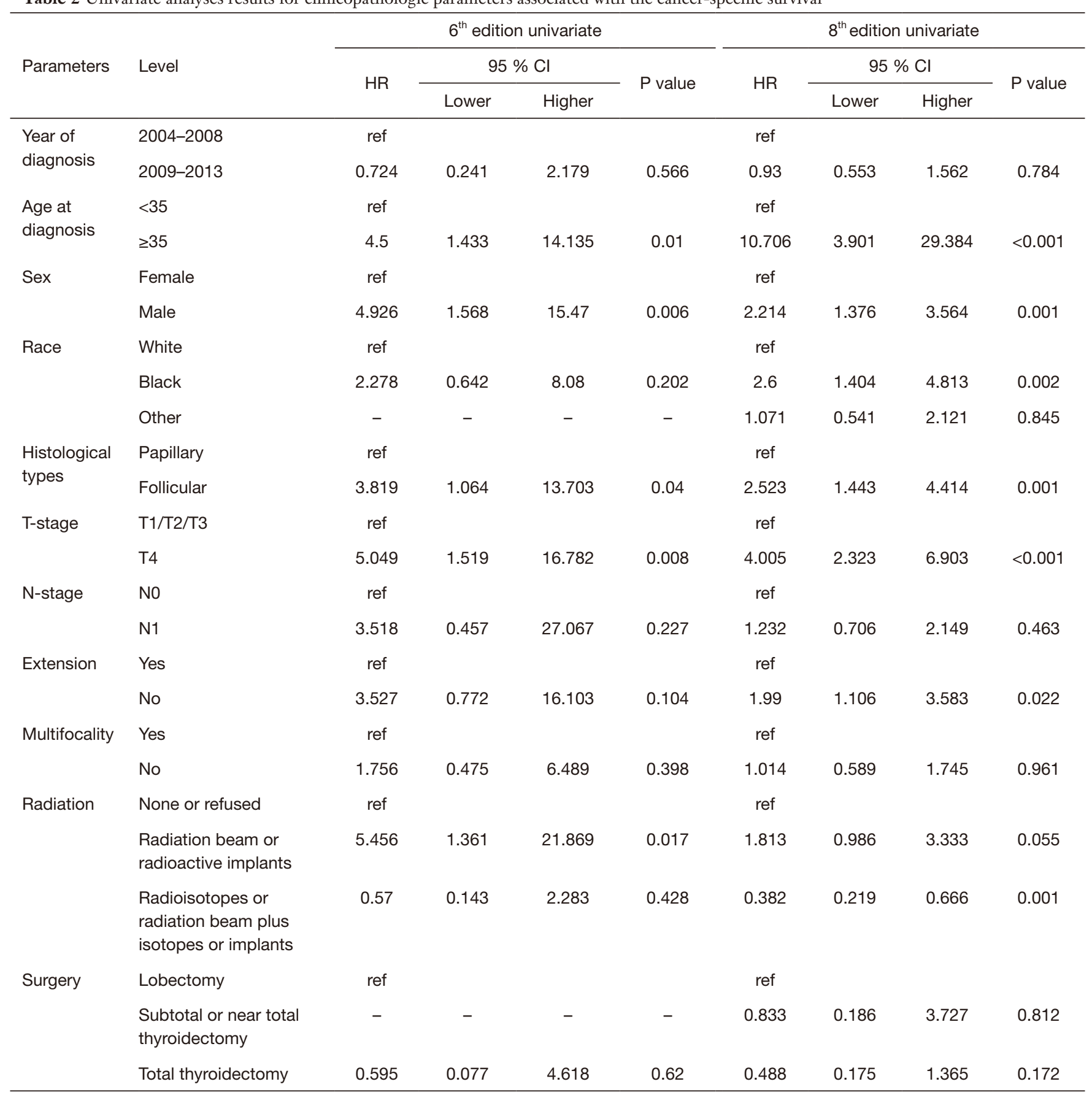


Table 3 Univariate analyses results for clinicopathologic parameters associated with the overall survival

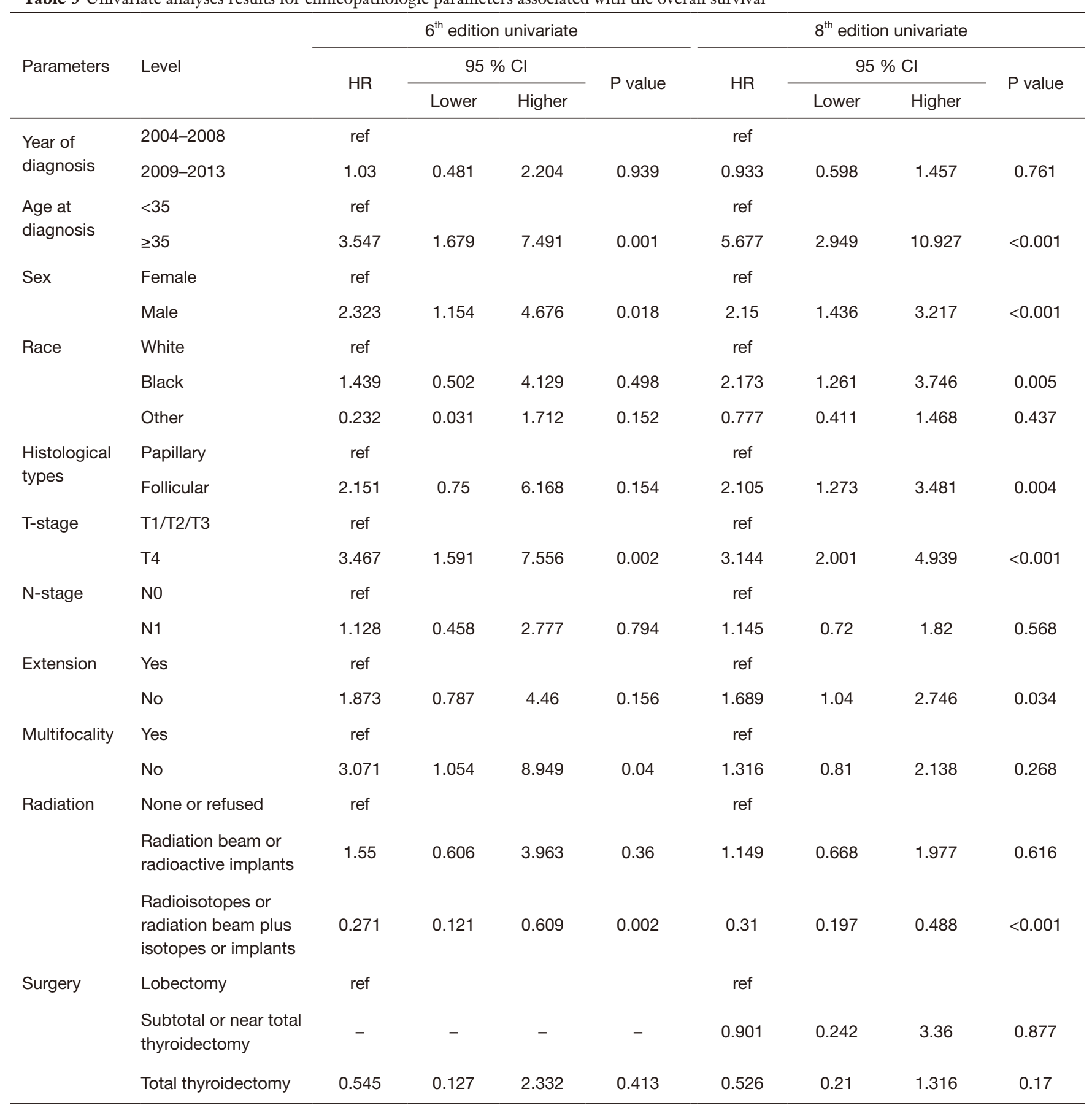


Table 4 Multivariate analyses results for clinicopathologic parameters associated with the cancer-specific survival in 8.0-based analysis

\begin{tabular}{|c|c|c|c|c|c|}
\hline \multirow{3}{*}{ Parameters } & \multirow{3}{*}{ Level } & \multicolumn{4}{|c|}{$8^{\text {th }}$ edition multivariate } \\
\hline & & \multirow{2}{*}{$\mathrm{HR}$} & \multicolumn{2}{|c|}{$95 \% \mathrm{Cl}$} & \multirow{2}{*}{$P$ value } \\
\hline & & & Lower & Higher & \\
\hline \multirow[t]{2}{*}{ Year of diagnosis } & 2004-2008 & ref & & & \\
\hline & 2009-2013 & 1.174 & 0.58 & 2.377 & 0.656 \\
\hline Age at diagnosis & $\geq 35$ & 34.06 & 4.493 & 258.208 & 0.001 \\
\hline \multirow[t]{2}{*}{ Sex } & Female & ref & & & \\
\hline & Male & 1.885 & 0.962 & 3.696 & 0.065 \\
\hline Race & White & ref & & & \\
\hline \multirow[t]{2}{*}{ Histological types } & Papillary & ref & & & \\
\hline & Follicular & 1.862 & 0.745 & 4.655 & 0.183 \\
\hline \multirow[t]{2}{*}{ T-stage } & $\mathrm{T} 1 / \mathrm{T} 2 / \mathrm{T} 3$ & ref & & & \\
\hline & $\mathrm{T} 4$ & 1.951 & 0.835 & 4.555 & 0.123 \\
\hline \multirow[t]{2}{*}{$\mathrm{N}$-stage } & No & ref & & & \\
\hline & $\mathrm{N} 1$ & 2.132 & 0.861 & 5.277 & 0.102 \\
\hline \multirow[t]{2}{*}{ Extension } & Yes & ref & & & \\
\hline & No & 1.34 & 0.488 & 3.682 & 0.57 \\
\hline Radiation & $\begin{array}{l}\text { Radioisotopes or radiation beam } \\
\text { plus isotopes or implants }\end{array}$ & 0.858 & 0.389 & 1.891 & 0.704 \\
\hline \multirow[t]{3}{*}{ Surgery } & Lobectomy & ref & & & \\
\hline & $\begin{array}{l}\text { Subtotal or near total } \\
\text { thyroidectomy }\end{array}$ & 0.644 & 0.113 & 3.653 & 0.619 \\
\hline & Total thyroidectomy & 0.273 & 0.073 & 1.022 & 0.054 \\
\hline
\end{tabular}


Table 5 Multivariate analyses results for clinicopathologic parameters associated with the overall survival

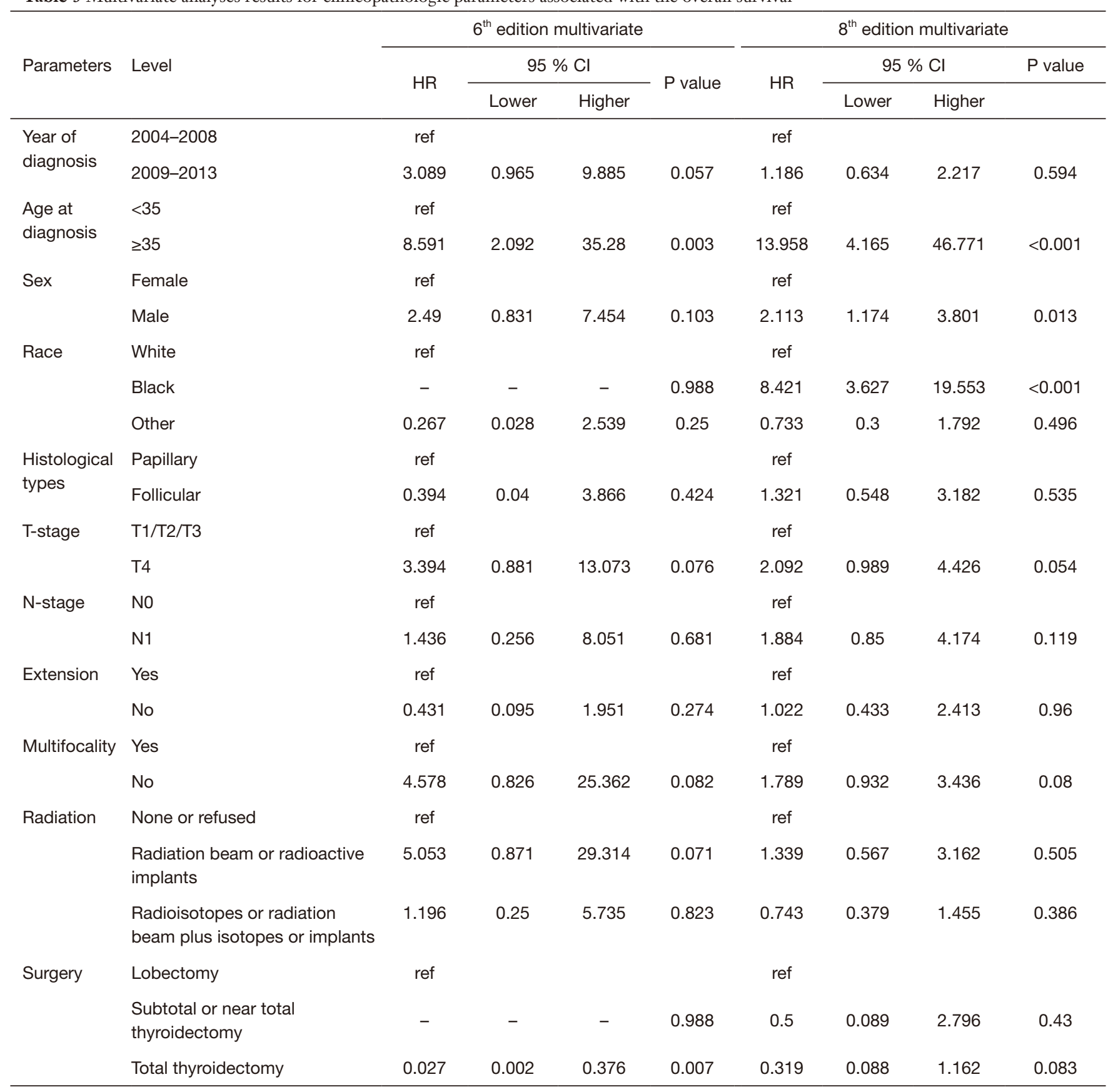

Table 6 Cancer-specific mortality and all-cause mortality for the cancer specific deaths and all cause deaths of thyroid cancer in 6.0- and 8.0-based analysis

\begin{tabular}{|c|c|c|c|c|c|c|c|c|}
\hline Extension & \multicolumn{2}{|c|}{ Cancer-specific mortality } & \multicolumn{2}{|c|}{ Cancer-specific mortality } & \multicolumn{2}{|c|}{ All-cause mortality } & \multicolumn{2}{|c|}{ All-cause mortality } \\
\hline $6^{\text {th }}$ edition & 15 & 5.40 & 11.55 & $6.843-19.508$ & 32 & 11.60 & 24.76 & $17.310-35.4096$ \\
\hline $8^{\text {th }}$ edition & 70 & 15.20 & 37.24 & $29.314-47.321$ & 98 & 21.30 & 51.70 & $42.190-63.349$ \\
\hline
\end{tabular}


Table 7 Cancer-specific mortality and all-cause mortality of several relevant factors for the cancer specific deaths and all cause deaths of thyroid cancer in 6.0- and 8.0-based analysis

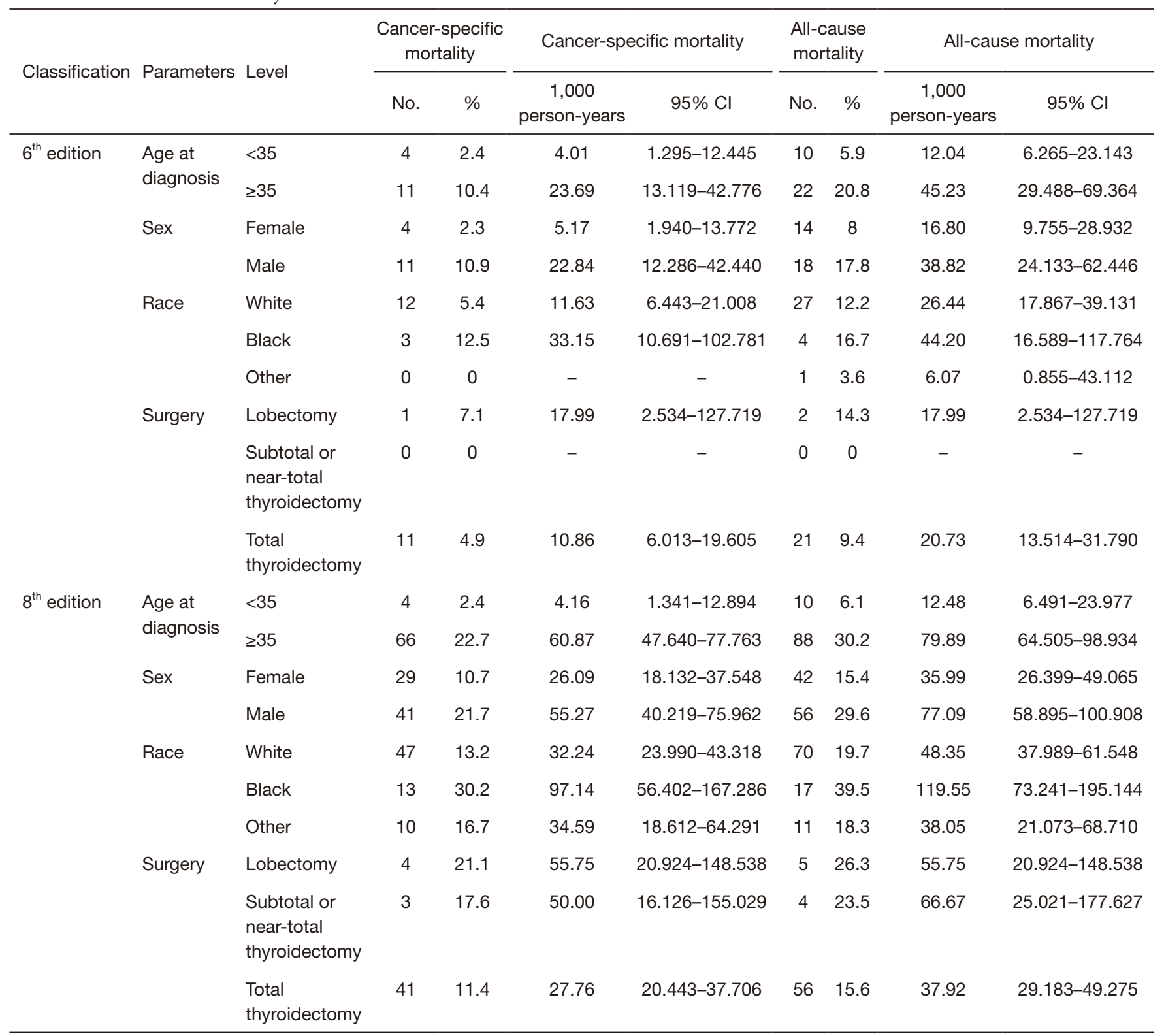

for more than $90 \%$ of the nearly 50,000 cases of thyroid cancers diagnosed in the US every year (7). Age is known to be an important prognostic factor for DTC patients, although far less attention has been paid to younger patients owing to their better survival relative to older patients. However, thyroid cancer has become the most common cancer in 16-33-year-old American individuals. Despite this increase in thyroid cancer incidence among young patients, there are many clinical challenges facing this group in terms of diagnosis, reduced healthcare access, and inconsistent care. Thus, this population requires closer attention in regard to the resolution of the challenges hindering favorable patient outcomes (10). Although DTC is a disease with a generally good outcome, patients presenting with distant metastatic disease have less favorable outcomes (14). Poor survival in patients with DTC that present with metastatic disease has been demonstrated previously and is not disputed (15). Thus, we conducted a subgroup analysis 

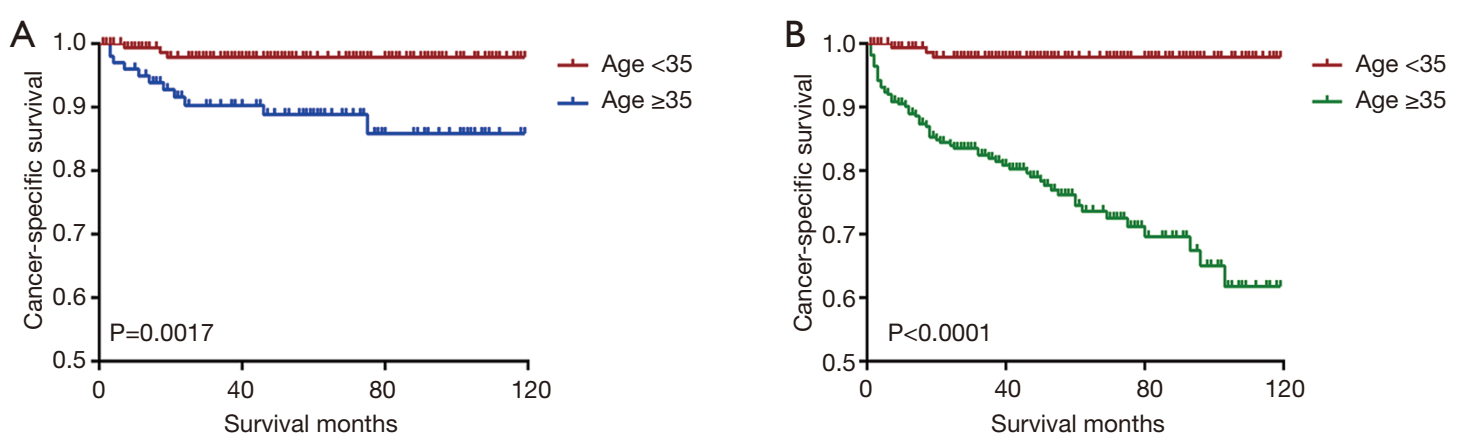

Figure 2 Kaplan Meier curves for cancer-specific survival among patients stratified by age for 6.0-based analysis (A) and 8.0-based analysis (B).
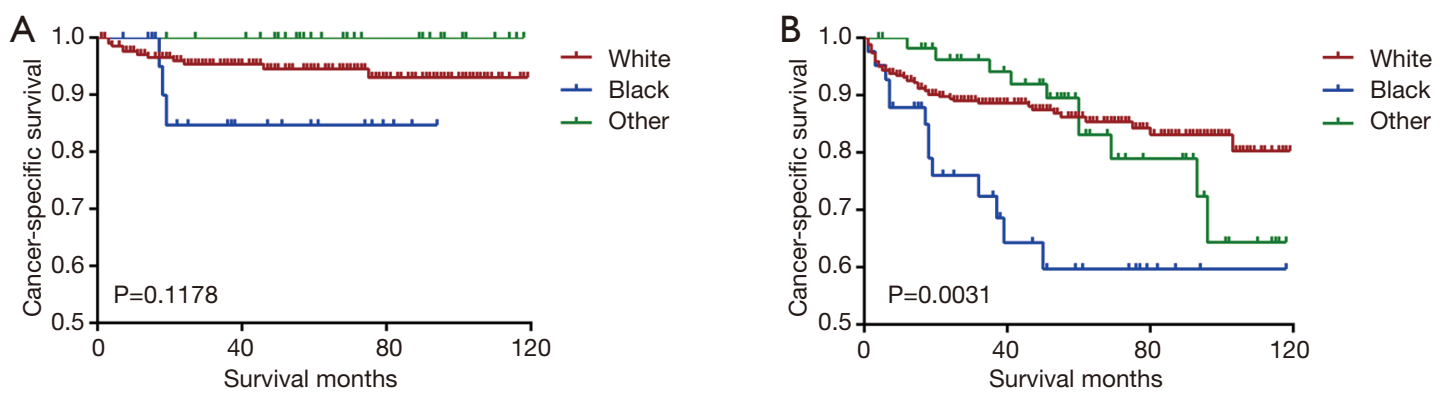

Figure 3 Kaplan Meier curves for cancer-specific survival among patients stratified by race for 6.0-based analysis (A) and 8.0-based analysis (B).
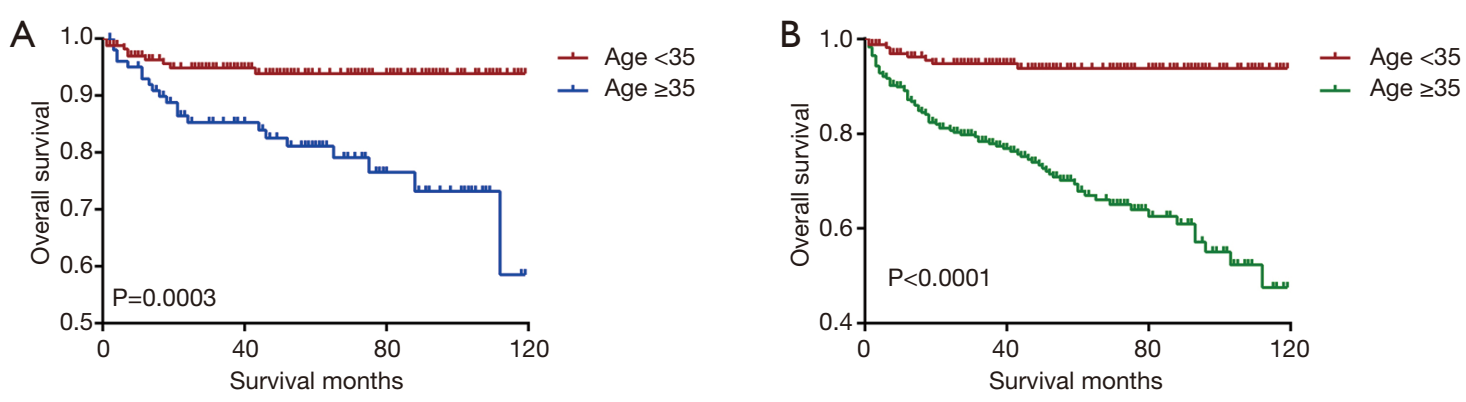

Figure 4 Kaplan Meier curves for overall survival among patients stratified by age for 6.0-based analysis (A) and 8.0-based analysis (B).

on a series of patients who were under the cut-off age and in stage II.

The current staging system for DTCs emphasizes the importance of patient age to the prognosis of these cancers by creating two dichotomous patient groups based on an assigned cut-off of age (13). Age first appeared as a prognostic factor in the second edition of the AJCC Cancer Staging Manual published in 1983, in which patients were grouped according to an age of less than 45 years or above 45 years; this grouping system was maintained until publication of the $8^{\text {th }}$ edition (16). Using an evidenced- based approach, a multidisciplinary committee of thyroid cancer experts made several substantial modifications to the seventh edition of the AJCC/TNM staging system that are now embodied in the new eighth edition staging system, including changing the cut-off point for age in the staging system from 45 to 55 years. According to this system, all patients younger than 55 years of age are considered to have stage I disease (regardless of tumor size, lymph node status, histologic subtype, or the presence/absence of extrathyroidal extension) unless they have distant metastases, in which case their disease is considered to be at stage II (9). In order 

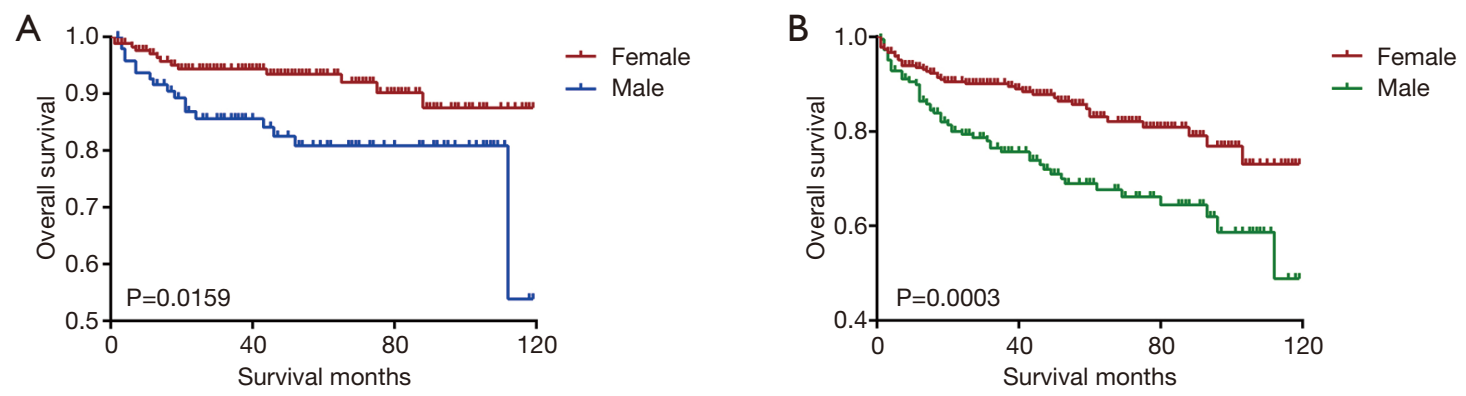

Figure 5 Kaplan Meier curves for overall survival among patients stratified by sex for 6.0-based analysis (A) and 8.0-based analysis (B).
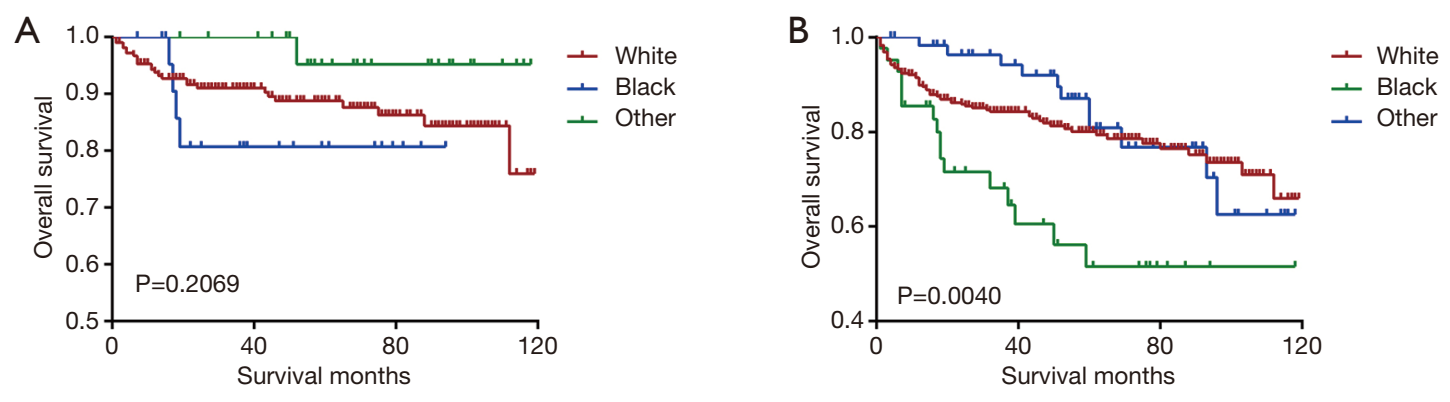

Figure 6 Kaplan Meier curves for overall survival among patients stratified by race for 6.0-based analysis (A) and 8.0-based analysis (B).
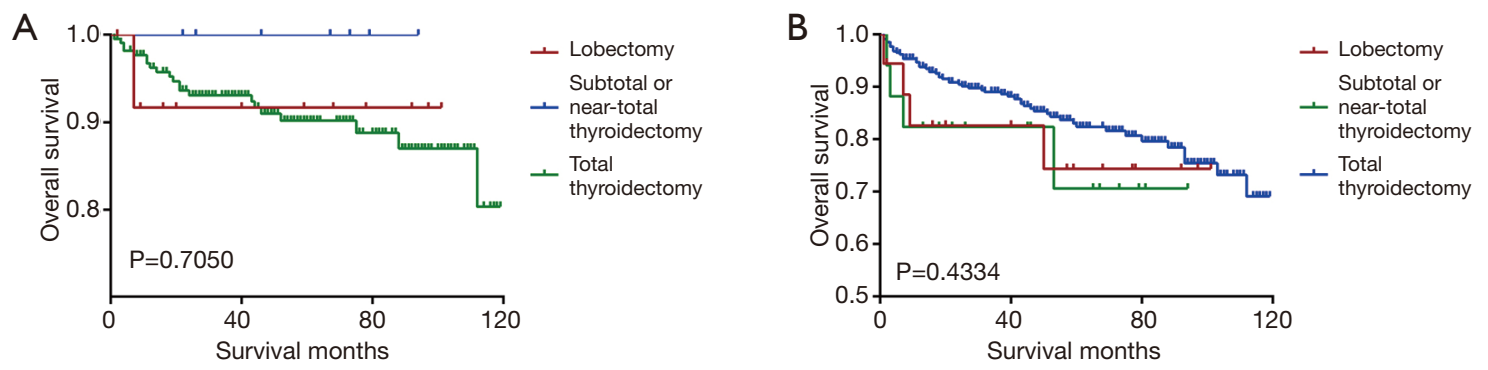

Figure 7 Kaplan Meier curves for overall survival among patients stratified by surgery for 6.0-based analysis (A) and 8.0-based analysis (B).

to explore the differences between the application of these two versions of the standard, we applied the cut-off ages for both staging systems in our subgroup analysis, which revealed similar proportions of stage II patients under both criteria.

Our Kaplan-Meier analyses showed a significant difference in DTC-specific and patient survival curves between patients below and above 35 years old under both AJCC 6.0- and 8.0-based analyses. Overall, regardless of the edition the standard was based on, patients $\geq 35$ years old had worse outcomes than patients $<35$ years old for both CSS and OS, which is consistent with the results of Oyer et al. (13) Moreover, it is noteworthy that both the cancerspecific and all-cause mortality rates per 1,000 person-years of patients aged $\geq 35$ years old remarkably differed from those of patients $<35$ years old. Tran Cao et al. (2). proposed that under the current AJCC staging guidelines for DTC, the protective effects of age may be overestimated, especially in the setting of metastatic disease, resulting in the under-staging of young patients. Thus, further detailed investigations are needed to determine whether the age cutoff point in the staging system for young patients should be refined.

Nilubol et al. (17) reported that male patients are more 
likely to have more advanced disease; however, they did not identify sex as an independent prognostic factor for DSS. Jonklaas et al. (15) conducted an in-depth analysis of the impact of age and sex on survival in patients with PTC and noted that, among younger patients, females had better outcomes ( $<55$ years). In our subgroup analysis, sex emerged as a risk factor for OS in young patients at stage II: being male was associated with a statistically significant increase in overall mortality according to both the AJCC 6.0- and 8.0-based analyses. However, race only emerged as a risk factor for CSS and OS when we applied the $8^{\text {th }}$ edition criteria, while the result for the AJCC 6.0-based analysis showed the reverse pattern. Thus, the effect of race was associated with the classification accuracy of age, indicating the need for additional studies to determine whether race should be considered a risk factor for young patients with stage II DTC based on the $8^{\text {th }}$ edition AJCC staging system.

It must also be noted that databases such as the SEER registry have inherent limitations that should be taken into consideration when interpreting our results. First, we were unable to distinguish between patients at $\mathrm{T} 1 / \mathrm{T} 2 / \mathrm{T} 3$ stages in the 8.0-based analysis because of coding overlaps. Thus, we used a unified standard, defining patients in the T1/ $\mathrm{T} 2 / \mathrm{T} 3$ stages as a single group in the analyses based on both editions. Furthermore, information regarding family history, vascular invasion, or other histologic findings was not evaluated or included in our dataset. Third, we did not account for recurrence and surgery-related comorbidities in our analyses. The SEER database does not include information regarding whether the patients underwent repeated surgery, and this lack of information may have biased our findings.

Despite these several inherent weaknesses of the use of national retrospective databases, the strengths of the present work are derived from the fact that the population data were gathered across varied regions and hospital settings. As the study period spans several decades, the workup and management of patients with regional and distant metastases undoubtedly changed over this time. To account for these chronological trends as potential confounders, the exact year of diagnosis should be incorporated into the multivariate model to account for changes in practice patterns over time.

\section{Conclusions}

In summary, our analyses demonstrate that age is a risk factor for CSS and OS in young patients with stage II
DTC, and that young male patients appear to have poorer survival than young female patients. Race also appears to be a potential risk factor for young patients in stage II, which may lead to new perspectives and improvements in applying both the older and newer versions of the AJCC staging system; however, additional evidence is required to validate these results.

\section{Acknowledgments}

Funding: None.

\section{Footnote}

Reporting Checklist: The authors have completed the STROBE reporting checklist. Available at http://dx.doi. org/10.21037/gs-20-46

Conflicts of Interest: All authors have completed the ICMJE uniform disclosure form (available at http://dx.doi. org/10.21037/gs-20-46). The authors have no conflicts of interest to declare.

Etbical Statement: The authors are accountable for all aspects of the work in ensuring that questions related to the accuracy or integrity of any part of the work are appropriately investigated and resolved. And this study conformed to the provisions of the Declaration of Helsinki (as revised in 2013). The study cohort of patients with DTC was recruited from the SEER database, which is supported by the National Cancer Institute and Centers for Disease Control and Prevention. Since SEER is a publicly available database with anonymized data, no ethical review was required.

Open Access Statement: This is an Open Access article distributed in accordance with the Creative Commons Attribution-NonCommercial-NoDerivs 4.0 International License (CC BY-NC-ND 4.0), which permits the noncommercial replication and distribution of the article with the strict proviso that no changes or edits are made and the original work is properly cited (including links to both the formal publication through the relevant DOI and the license). See: https://creativecommons.org/licenses/by-nc-nd/4.0/.

\section{References}

1. Konstantinidis A, Tracy E, Sosa JA, et al. Risk prediction 
in children and adults less than 45 years old with papillary thyroid cancer. Expert Rev Endocrinol Metab 2017;12:355-65.

2. Tran Cao HS, Johnston LE, Chang DC, et al. A critical analysis of the American Joint Committee on Cancer (AJCC) staging system for differentiated thyroid carcinoma in young patients on the basis of the Surveillance, Epidemiology, and End Results (SEER) registry. Surgery 2012;152:145-51.

3. Aschebrook-Kilfoy B, Kaplan EL, Chiu BC, et al. The acceleration in papillary thyroid cancer incidence rates is similar among racial and ethnic groups in the United States. Ann Surg Oncol 2013;20:2746-53.

4. Davies L, Welch HG. Increasing incidence of thyroid cancer in the United States, 1973-2002. JAMA 2006;295:2164-7.

5. Cady B, Rossi R. An expanded view of risk-group definition in differentiated thyroid carcinoma. Surgery 1988;104:947-53.

6. Hay ID, Grant CS, Taylor WF, et al. Ipsilateral lobectomy versus bilateral lobar resection in papillary thyroid carcinoma: a retrospective analysis of surgical outcome using a novel prognostic scoring system. Surgery 1987;102:1088-95.

7. Orosco RK, Hussain T, Brumund KT, et al. Analysis of age and disease status as predictors of thyroid cancer-specific mortality using the Surveillance, Epidemiology, and End Results database. Thyroid 2015;25:125-32.

8. Nixon IJ, Wang LY, Migliacci JC, et al. An International Multi-Institutional Validation of Age 55 Years as a Cutoff for Risk Stratification in the AJCC/UICC Staging System for Well-Differentiated Thyroid Cancer. Thyroid

Cite this article as: Huang $\mathrm{Y}$, Zhou L, Zeng W, Chen S, Zhou W, Wei W, Zhang C, Hu D, Wang M, Liu Z, Guo L. Novel analysis of prognosis of young patients with stage II differentiated thyroid cancer based on AJCC 8.0 and 6.0 criteria to implement the staging system. Gland Surg 2020;9(5):1244-1257. doi: $10.21037 /$ gs-20-46
2016;26:373-80.

9. Perrier ND, Brierley JD, Tuttle RM. Differentiated and anaplastic thyroid carcinoma: Major changes in the American Joint Committee on Cancer eighth edition cancer staging manual. CA Cancer J Clin 2018;68:55-63.

10. Araque DVP, Bleyer A, Brito JP. Thyroid cancer in adolescents and young adults. Future Oncol 2017;13:1253-61.

11. Liu Z, Zhao Q, Zeng W, et al. Prognosis of a rare subtype of thyroid cancer: Spindle cell thyroid carcinoma. Medicine (Baltimore) 2018;97:e13053.

12. Liu Y, Liu Z, Zhao Q, et al. Propensity score matching analysis of the prognosis for the rare insular subtype of thyroid cancer based on SEER database. Oncotarget 2017;8:101623-33.

13. Oyer SL, Smith VA, Lentsch EJ. Reevaluating the prognostic significance of age in differentiated thyroid cancer. Otolaryngol Head Neck Surg 2012;147:221-6.

14. Nixon IJ, Whitcher MM, Palmer FL, et al. The impact of distant metastases at presentation on prognosis in patients with differentiated carcinoma of the thyroid gland. Thyroid 2012;22:884-9.

15. Jonklaas J, Nogueras-Gonzalez G, Munsell M, et al. The impact of age and gender on papillary thyroid cancer survival. J Clin Endocrinol Metab 2012;97:E878-E887.

16. Beahrs OH, Myers MH. editors. American Joint Committee on Cancer Manual for Staging of Cancer. 2nd ed. Philadelphia, PA: J.B. Lippincott; 1983.

17. Nilubol N, Zhang L, Kebebew E. Multivariate analysis of the relationship between male sex, disease-specific survival, and features of tumor aggressiveness in thyroid cancer of follicular cell origin. Thyroid 2013;23:695-702. 


\section{Materials and methods}

Here is some summaries about these process.

(I) Restaging of the cases of DTC in the data of SEER 18 from 2004 to 2013 was performed using the criteria of AJCC 8.0. For patients under 55 years old, stage can be assigned directly according to the M-status. But for patients older than 55 years which are at T3 in AJCC 6th edition, their T categories need to re-assigned. If the tumor size is larger than $4 \mathrm{~cm}$ or patients have lymph node metastasis, it will be assigned stage II. If this patient has distant metastasis, it will be assigned stage IVB. For the patients, whose tumor size $\leq 4 \mathrm{~cm}$, and no lymph node and distant metastasis, it is hard to identifying whether minimum or gross extra thyroid extension exists in these patients (T1/T3 or T2/T3), therefore it is hard to assigned the stage (I or II). There patients will be excluded for our analysis.

(II) Additionally, 209 patients were found with T0, whose tumor size is 0 . But some of them have lymph node metastasis or even distant metastasis. If the age $<55$, it is easy to identify the stage I or II based on the M1 status. For patients with age $\geq 55$, if patients have lymph node metastasis, it will be assigned stage II. If this patient has distant metastasis, it will be assigned stage IVB. But if the patient is T0, N0/NX and M0, it is hard to identify the stage, here I used 'unknown stage'.

(III) At the same time, 3,958 patients were found with TX, whose tumor size is unknown or thyroid extension is unknown. If the age $<55$, it is easy to identify the stage I or II based on the M1 status. For patients with age $\geq 55$, if this patient has distant metastasis M1, it will be assigned stage IVB. But for others, it is hard to identify the stage without the T category (Stage I, II, III or IVA), and here I used 'unknown stage'.

(IV) Also, 45 patients were found at T4, but could not identifying whether T4a or T4b. If the age $<55$, it is easy to identify the stage I or II based on the M1 status. For patients with age $\geq 55$, if this patient has distant metastasis M1, it will be assigned stage IVB. But for others, it is hard to identify the stage (III or IVA), and here I used 'unknown stage'.

(V) Finally, 3,983 patients are 'unknown stage' patients, and 95,032 patients have new AJCC 8th edition stage information.

Table S1 Characteristics of patients with differentiated thyroid cancer from the Surveillance, Epidemiology, and End Results Database (SEER) [2004-2013] stratified by 6.0 and 8.0 edition AJCC staging system

\begin{tabular}{|c|c|c|c|c|}
\hline Patient characteristics & \multicolumn{2}{|c|}{ AJCC Version 6} & \multicolumn{2}{|c|}{ AJCC Version 8} \\
\hline \multicolumn{5}{|l|}{ Sex } \\
\hline Female & 72,135 & 77.04 & 72,928 & 76.95 \\
\hline Male & 21,504 & 22.96 & 21,847 & 23.05 \\
\hline$<45 / 55^{a}$ & 37,144 & 39.67 & 62,019 & 65.44 \\
\hline$\geq 45 / 55^{a}$ & 56,495 & 60.33 & 32,756 & 34.56 \\
\hline \multicolumn{5}{|l|}{ AJCC stage at diagnosis } \\
\hline I & 68,600 & 73.26 & 84,828 & 89.5 \\
\hline IV & 6,283 & 6.71 & 1,679 & 1.77 \\
\hline
\end{tabular}

a, 45 years old in the 6 th edition analysis and 55 years old in 8th edition analysis, respectively. 
Table S2 Differentiated thyroid cancer-specific deaths and hazard ratios of mortality in patients with different disease stages stratified by 6.0 and 8.0 edition AJCC staging system

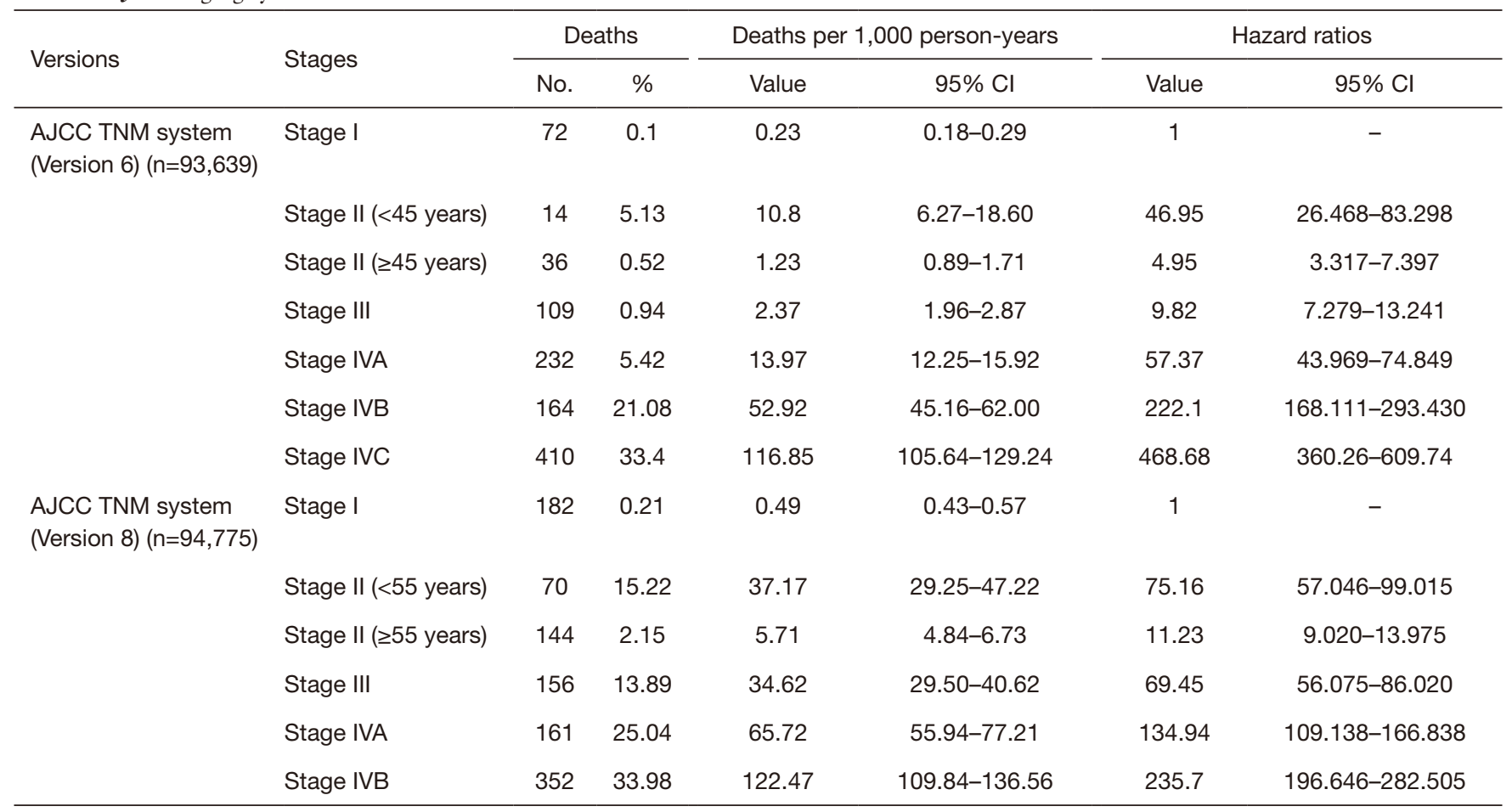


Table S3 Multivariate analyses results for clinicopathologic parameters associated with the cancer-specific survival in 6.0-based analysis

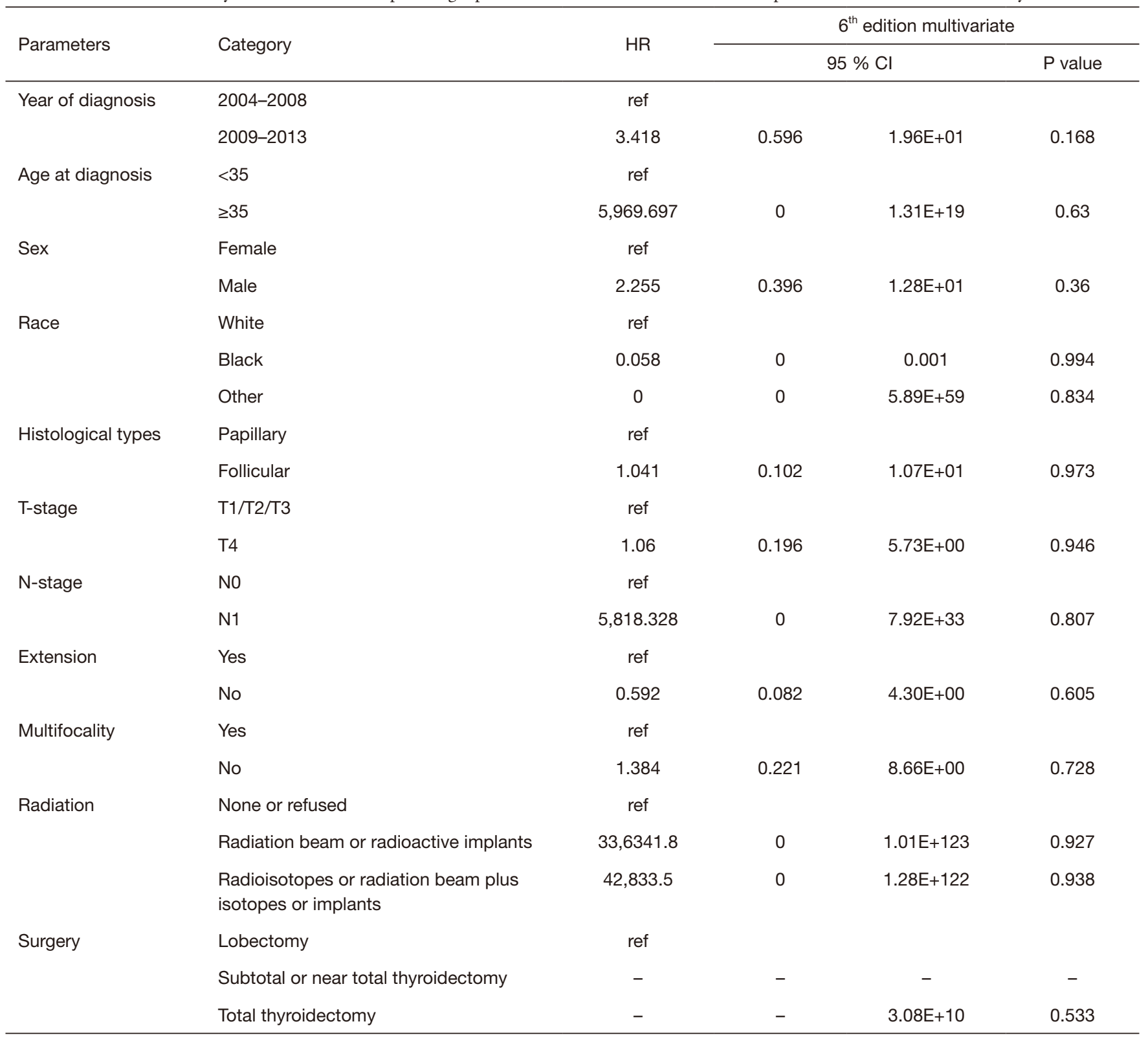

